\title{
About Clinical Heterogeneity of FSHD with Some Historical Remarks
}

\section{Kazakov VM*}

Department of Neurology, Pavlov State Medical University, St. Petersburg, Russia

What is the (facio) scapuloperoneal muscular dystrophy? Is it an independent form or a variant of FSHD? Short extracts from the works of some authors given below specify their position concerning nosological place of (facio) scapuloperoneal muscular dystrophy.

Oransky [1] considered that it is a peculiar autosomal dominant form of muscular dystrophy with predominantly early involvement of the shoulder - girdle and peroneal group muscles and in some cases with a mild weakness of the face and trunk muscles.

Davidenkov and Kulkova [2] "Scapulo-peroneal amyotrophy is probably an independent disease which significantly closer to the Landouzy-Dejerine amyotrophy" and later... "It is possible to suppose that actually scapula-peroneal amyotrophy and Landouzy-Dejerine amyotrophy are connected with the action of the same basic gene but different phenotypes may be due to a modified action of the changing familial gene pool.."

Seitz [3] related scapuloperoneal myogenic syndrome to Erb's Dystrophia musculorum progressiva.

Davidenkov and Dogel [4] “...Scapuloperoneal amyotrophy is a well outlined, peculiar form which obviously belongs to a combined group of myopathies and approaches mostly to the amyotrophy of LandouzyDejerine, although it has some features which differ substantially both from the classical amyotrophy of Landouzy-Dejerine and from the other variants of myopathy."

Becker [5] "Autosomal dominant myogenic scapulo-peroneal syndrome probably is an independent form of muscular dystrophy."

Ricker and Mertens [6] "The myogenic autosomal dominant scapulo-peroneal syndrome is a variant of FSHD."

Zellweger and McCormick [7] "Should the familial disorder reported by Oransky be considered as scapuloperoneal dystrophy, one would need to postulate a new type of autosomal dominant muscular dystrophy which would be classified between the scapulohumeral variant (Erb) of type I (Landouzy-Dejerine) and autosomal dominant, distal type of Welander."

Serratrice et al. [8] wrote that a scapula-peroneal muscular atrophy "It is probably an unusual form of progressive muscle atrophy of facioscapulo-humeral type with peroneal involvement - or myopathies (facio) scapuloperoneal"

Hausmanova-Petrusewicz [9] "Scapuloperoneal syndrome (its myopathic variant) appears to be a variety of the FSHD."

Kazakov [10], Kazakov et al. [11,12] “...the FSHD type can be considered as an independent form. The best name for it is "facio-scapulo-limb (FSL) muscular dystrophy adding the eponym "Duchenne-Landouzy-Dejerine". FSL myodystrophy is clinically heterogeneous and two varieties in the form of muscular dystrophy included: the gradually descending variety and the more frequently a descending one, characterized by a "jump" from the upper part of the body to the peroneal groups of the shin muscles (the descending variety with a "jump"). Genealogical analysis of 62 families suggests the existence of a clinical and genetic heterogeneity in FSL myodystrophy".

Thomas et al. [13] "The clinical features of the present series (a scapuloperoneal myopathy), however, clearly indicate that they represent a distinct group."
Carroll [14] "....Although the preceding paragraphs form the basis of the name FSHD, other muscles are often involved... Also sometimes what it noted early in the disease is weakness of the anterior tibial muscles Walton and Gardner-Medwin [15,16] In our experience, this has been a frequent occurrence. .... Thus, if a patient were encountered with scapular muscle and anterior tibial weakness along with very little facial weakness, then the term scapuloperoneal dystrophy would just as well apply."

(Serratrice et al. [17]; Kazakov et al. [11]) "Autosomal dominant scapuloperoneal myopathy.... The possibility that some recorded cases have "incomplete" or abortive forms of facioscapulohumeral muscular dystrophy is suggested by the large kindred reported by Kazakov et al. in which facial muscles was a late and mild feature in many cases."

Serratrice et al. [17] “...1) scapula-peroneal dystrophies of a predominantly hereditary nature (31 cases) The most frequently observed form (very probably of a dystrophic nature) appear to be only a topographical variety of Landouzy-Dejerine's facio-scapulo-humeral myopathy, particularly as the facial region is often involved. The following forms...: pure facio-scapulo-humeral, facio-scapulo-humeral with peroneal involvement, facio-scapulo-peroneal, and pure scapulaperoneal."

Padberg [18] “...Ricker and Mertens did one year earlier (1968) namely, that a scapuloperoneal syndrome might be a stage in the development of FSHD. A similar conclusion was drawn by Kazakov et al. [11] about the famous family K. This family was reported initially by Oransky in 1927. ...Kazakov et al. [11] reexamined this family... In his opinion this family suffered from FSHD" and late "... autosomal dominant SP myopathy with facial weakness in indistinct from FSHD" (My note: this citation is not exact. I wrote that in the famous kindred $\mathrm{K}$. there is a FSLD descending with a "jump" type, but not a classical FSHD (or FSLD gradually descending type).

Rossi et al. [19] "We agree with Kazakov et al. [11] that involvement of the peroneal muscles could be a stage in the development of Landouzy-Dejerine dystrophy rather than characterizing a separate entity as Becker proposed [5]". \{(My note: this citation is not exact)\}.

Brooke [20] "Scapuloperoneal dystrophy is probably a variety of facioscapulohumeral dystrophy.... Bilateral foot-drop may be present (on FSHD). Occasionally this is one of the initial symptoms and it is then difficult to know whether to call the disorder facioscapulohumeral dystrophy or scapuloperoneal dystrophy."

McKusick [21] "Kazakov et al. provided a follow-up the kindred reported by Davidenkow (1939). The disorder in many ways resembled Landouzy-Dejerine facioscapulohumeral muscular dystrophy."

*Corresponding author: Valery Kazakov, Department of Neurology, Pavlov State Medical University, L. Tolstoy Str. 6/8, 197022 St. Petersburg, Russia, E-mail: valerykazakov@mail.ru

Received March 26, 2013; Accepted May 14, 2013; Published May 18, 2013 Citation: Kazakov VM (2013) About Clinical Heterogeneity of FSHD with Some Historical Remarks. Hereditary Genetics S1: 006. doi:10.4172/2161-1041.S1-006

Copyright: @ 2013 Kazakov VM. This is an open-access article distributed under the terms of the Creative Commons Attribution License, which permits unrestricted use, distribution, and reproduction in any medium, provided the original author and source are credited. 
Swash and Schwartz [22] "The extensor digitorum brevis muscles may be spared both in FSHD and in scapulo-peroneal muscular dystrophy and the two disorders are probably closed related, if they are not variations of the same disorder."

In classification compiled by [23] the scapuloperoneal muscular dystrophy was presented as nosological entity: «c) Scapuloperoneal muscular dystrophy, autosomal dominant».

Munsat and Serratrice [24] "The scapuloperoneal dystrophies of autosomal dominant inheritance or sporadic... suggested a relationship between FSHD and scapuloperoneal syndrome. There appears to be a continuum with 4 clinical forms: pure FSHD; FSHD with peroneal weakness; scapuloperoneal dystrophy with facial weakness (in these cases, humeral muscles are frequently spared and ankle contractures are constant) and pure scapuloperoneal dystrophy."

Brouwer [25] "For instance, Oransky [1] described three families with autosomal dominant scapuloperoneal syndrome. .... Kazakov et al. reexamined the Oransky [1] family and finally concluded that this family suffered from FSHD". \{(My note: this citation is not exact) $\}$.

Jardine et al. [26] "The existence of scapuloperoneal myopathy, without contractures or cardiomyopathy, as a genetically separate condition from FSHD is uncertain.... Scapular onset muscular dystrophy without facial involvement inherited as an autosomal dominant condition, may be allelic with FSHD."

In classification compiled by Walton et al. [27] the scapuloperoneal muscular dystrophy was presented as nosological entity:

«c) Scapuloperoneal muscular dystrophy, autosomal dominant (not proven to differ from facioscapulohumeral muscular dystrophy; note possible neurogenic type)"

Kazakov [28] " $\ldots$ the FSHD is a heterogeneous form of muscular dystrophy. The name facio-scapulo-limb muscular dystrophy (FSLD) was offered for this disease. On the historical and clinical study FSLD may be divided into two nosological entities, namely: Facioscapulolimb muscular dystrophy, type 1 (FSLD1), a gradually descending type with initial facio-scapulo-humeral phenotype; autosomal dominant (Duchenne de Boulogne) and Facioscapulolimb muscular dystrophy, type 2 (FSLD2), a descending with a "jump" with initial FSP phenotype; autosomal dominant (Erb, Landouzy, Dejerine)."

Griggs et al. [29] "Kazakov et al. personally examined patients belonging to four generations of the family originally reported by Oransky .... and the clinical picture was indistinguishable from FSHD dystrophy". $\{($ My note: this citation is not exact $)\}$.

Tawil et al. [30] ..."Consequently, some authors suggest that a least the myopathic form of FSPs is a stage in the development of FSHD" (Kazakov et al.). $\{$ (My note, this citation is not exact)\}.

Rowland [31] "Locus heterogeneity... helped to explain many diseases ...such as the separation of scapuloperoneal muscular dystrophy from facioscapulohumeral dystrophy."

Wilhelmsen et al. [32] "If there were facial weakness in a person with autosomal dominant scapuloperoneal dystrophy, it would be indistinguishable from FSHMD. $\{$ (My note: this citation is not exact)\}.

Fisher and Upadhyaya [33] "Muscle disorders related to FSHD include scapuloperoneal and scapulohumeral dystrophies which are associated with minimal or absent facial weakness and may represent milder forms of the disease since there is evidence for involvement of the same genetic locus."
Bushby [34] "within the broad definition of FSHD there can be extreme variation... In some patients, muscle involvement remains restricted to the facial, scapular, and proximal upper limb musculature. In others, anterior tibial and peroneal muscles may be involved subsequently, leading to foot-drop as a relatively early sign. Alternatively, the proximal muscles of the lower limb may be involved before the distal muscles ..."

Padberg [35] "Subsequent nosography introduced the term scapuloperoneal [4] which is also the most concise summary of the early picture of FSHD patients with minimal facial weakness [1]. It took some time to recognize that most of the families reported to suffer from scapuloperoneal myopathy had FSHD [1]". \{(My note: this citation is not exact)\}.

Orrell and Griggs [36] "The status of scapuloperoneal syndrome is not fully defined. Clinical features overlap with FSHD... The availability of molecular diagnostic testing of FSHD has led to the reclassification of some patients previously diagnosed with scapuloperoneal syndrome as having FSHD."

Attarian et al. [37] "We propose that SP represents the mild end of the spectrum of FSHD with DFS ranged between 28 to $35 \mathrm{~kb}$."

The Neuromuscular Home Page (Washington University) (2003, 2012) [38] suppose "The scapuloperoneal weakness (scapular winging, foot dorsiflexion weakness and abdominal muscle weakness) observe on mild or atypical FSHMD1A, chromosome 4q35, dominant with small deletion $>35 \mathrm{~kb}$ DNA fragment".

Padberg [39]"However, it is likely that the adjective FSH later precluded recognition of the disease in some families with lower limb involvement $[1,13]$ while mild facial weakness in other families prompted the use of the term scapuloperoneal muscular dystrophy [2] My note: this citation is not exact (see below) $\}$. In any case, a discussion was necessary to get early foot-extensor weakness accepted as part of the natural course of FSHD [18].

In other articles \{Zeevaert et al. [40], Pou-Serradell [41]\} was written again about the wide clinical spectrum of $4 \mathrm{q} 35$ linked FSHD and the difficulty to diagnose unusual familial cases with facial-sparing and scapuloperoneal muscle affection related to $4 \mathrm{q} 35$ chromosomal deletion.

The question is: What is the autosomal dominant scapuloperoneal muscular dystrophy with minimal affection of facial muscles linked with $4 \mathrm{q} 35$ chromosomal deletion?

In the article published in Eur. Neurol [42] which by Padberg [18], Brouwer [25], Griggs et al. [29], Tawil et al. [30] and Wilhelmsen et al. [32] did not cited correctly enough (see above), I wrote in conclusion :"The study of this form of muscular dystrophy (facio-scapulo-limb or facioscapulohumeral) carried out by us, revealed the existence of two independent varieties: a gradually descending variety and more frequent a descending one with a "jump" of the atrophies from facial and shoulder girdle muscles to the peroneal group of the shin muscles (the descending variety with a "jump"). To our mind, it is to the latter variety that the muscular dystrophy in the K. kindred should be related (p. 358)".

In other article published in Clinical Genetics [43] which by Rossi et al. [19], Walton and Gardner-Medwin [15] and McKusick [21] did not total cited correctly enough (see above), I wrote: "From the clinical and genetic data available at the present time, is seems that the muscular dystrophy in the K. kindred is one of the varieties (namely, a descending type with a "jump") of the facio-scapulo-limb (or facioscapulohumeral) muscular dystrophy (p.41)". 
Our present clinical and MRI data, as well as our earlier investigations (1969-2009), allows suggesting that FSP muscular dystrophy is probably an independent form with "hard" (stereotypical) static and dynamic pattern of muscle involvement and a mild course of the disease. The term "FSLD2 descending with a "jump" with initial FSP phenotype (Erb, Landouzy and Dejerine type)" would be more correct. The FSP or (F) SP phenotype constitutes merely a stage in the development of FSLD2.

I suppose that classical AD FSPMD (or FSLD2, a descending with a "jump" with initial FSP phenotype) is different (according specific static and dynamic pattern of muscle involvement as well as a mild degree affection of mimic muscles and mild course of the disease) from the classical AD FSHD which was called by me as a FSLD1, a gradually descending with initial FSH phenotype (Duchenne de Boulogne type) [28] and may be these diseases are connected with the various $4 \mathrm{q} 35$ chromosomal mutations [28,42-68].

I would like to present the photos of two different patients who are both called in the World literature as a classical and typical FSHD $[48,49]$.

This is my proband K.N. aged 63 (Figure 2) from famous family K. with typical FSLD2 descending with a "jump" type [42]. At the age of 18.5 he had a pure mild scapuloperoneal phenotype [1] which at the age of 30 (Figure 1) transferred in (facio)-scapulo-peroneal-(humeral) phenotype [2], and then at the age of 63 (Figure 2) it transferred in final severe (facio)-scapulo-peroneal-humero (biceps brachii, but not triceps ) - femoro (posterior thigh muscles, but not quadriceps) -gluteal (gluteus maximus muscles, but not gluteus medius) phenotype with increased the lumbar lordosis due to the severe weakness of abdominal and gluteus maximus muscles and slight atrophy of the right half of upper lip. At the age of 68 he could walk independently and climb the stairs (on 6 floors) with the aid of a stick and railing and he could walk with the help of a stick on long distances. In his a first cousin once removed aged 39 and a second cousin once removed aged 16 the 4q35 DFS $33 / 30 \mathrm{~kb}$ were found, and in other a first cousin once removed aged 34 the $4 \mathrm{q} 35$ DFS was $24 / 21$ (double digestion). They all were presymptomatics.

The Figure 3 was taken from Wikipedia, the Free Encyclopedia, 4 November 2011 (en.wikipedia.org). In my opinion this 27-year-old female have a typical FSLD1 gradually descending type with initial FSH phenotype with gradually extension of weakness and atrophy from the face and shoulder girdle to the upper arm, trunk, pelvic girdle and thigh muscles. She has increased lumbar lordosis due to the severe weakness of erector trunci muscle predominantly together with abdominal and pelvic girdle muscles.

\section{Conclusion}

It is necessary to remark that in the present time there is an opinion that FSHD is genetically heterogeneous, but clinically a homogeneous disease. "In recent years .... allowed the identification of the two forms of FSHD, the classical autosomal dominant FSHD type 1, and FSHD type 2 characterized by identical clinical phenotype but associated with a different (epi) genetic defect” and after "... FSHD1 and FSHD2 share a common pathophysiological pathway since they present an identical clinical phenotype and FSHD2 genetic defect, and that the FSHD2 gene may act as a modified for disease in FSHD1 families" [69].

On my opinion FSHD1 on clinical picture and molecular genetics may be corresponded to FSLD2 (a descending variety with a "jump") due to deletion of D4Z4 on chromosome 4q35. This form was described at the first time by Erb, Landouzy and Dejerine under different names in 1882-1885 years [28].

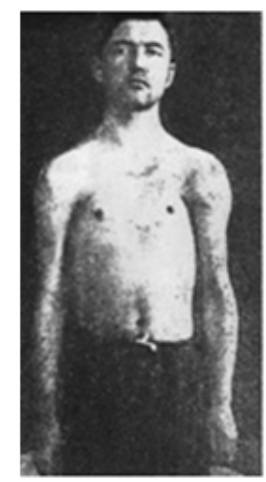

Figure 1: Transferred (facio)-scapulo-peroneal-(humeral) phenotype.

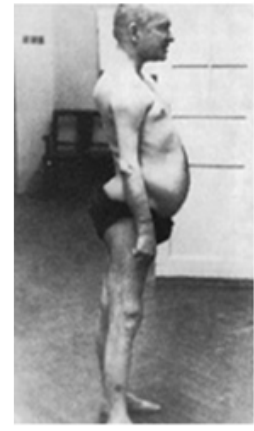

Figure 2: Typical FSLD2 descending with a "jump" type.

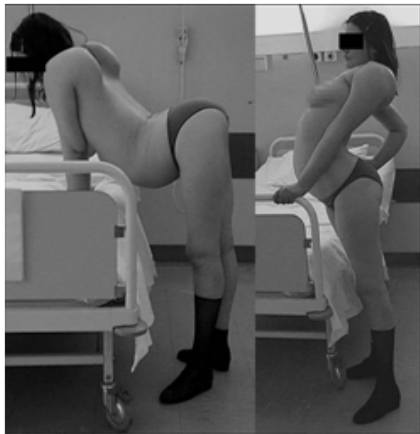

Figure 3: Typical FSLD1 gradually descending type with initial FSH phenotype.

Another form of FSHD which was called as a FSHD2 due to hypomethylation of D4Z4 on chromosome 4 and $10[69,70]$ and may be corresponded to FSLD1 (a gradually descending type) which was described at the first time by Duchenne in 1855 under the name "progressive hereditary muscular atrophy with fatty degeneration beginning with a face, trunk and limbs in adults or in youth or in children" [28]. That is why with historical point of view the FSHD1 more correctly to name as a FSHD2 but a FSHD2 - as a FSHD1. However, with molecular genetics point of view (if to accept in attention the time of molecular genetics description of these forms) the names FSHD1 and FSHD2 are correct.

In 1969-1971, I analyzed the pattern of muscle involvement (67 isolated muscles and their parts) in different stages of the disease of 200 patients with FSLD (or FSHD): 145 cases were taken from the 
World literature and 55 cases were under personal observation [10,11]. Between these 200 patients of 78 (31 personal cases and 47 from literature, 59 hereditary and 19 sporadic) had developed FSLD2 a descending with a jump type with the FSP or FSP $(\mathrm{H})$ phenotypes $(38$ patients) and with the final FSPFGH (facio-scapulo-peroneal-femoro (posterior thigh muscles)-gluteo (gluteus maximus)-humeral (biceps brachii) or the FSPHFG phenotypes (40 patients).

Besides this, between 200 analyzed FSLD (FSHD) cases of 60 (47 hereditary and 11 sporadic cases included Duchenne's cases, herteditary 8 and sporadic 3 taken from the World literature and only 2 my personal sporadic cases) had developed FSLD1 a gradually descending type $[10,11]$. Between these 60 cases the 31 were with the FSHGF (facio-scapulo-humero-gluteo-femoral) phenotypes and the 29 cases were with the final FSHGFP (facio-scapulo-humero-gluteofemoro-peroneal) phenotypes. In last group the pelvic girdle and thigh muscles were more severe affection then peroneal group excluded 6 cases in which the peroneal group muscles were the same severe affection [71-75].

It was found that only one of the two type of the disease (the gradually descending type or the descending one with a jump) occurred in each family. The distribution of the muscle affections in the members of the same family did not usually overstep the limits of the type of the development of the disease.

As well the existence of FSLD1 are confirm the fact that in some Handbooks on Muscle Diseases or Neurology the FSHD was described as a gradually descending muscular dystrophy with affection of pelvic girdle, trunk and hip muscles after involvement of the face, scapular and humeral muscles (and others).

Thus, in my opinion the FSLD1 and FSLD2 are clinical and historically well documented i.e. FSLD (or FSHD) is genetically as well as clinically a heterogeneous disease [10-12,28,42-46,49-71].

\section{References}

1. Oransky V (1927) Uber einen hereditaren Typus progressiver Muskeldystrophie. Dt Z NervHeilk 99: 147-55.

2. Davidenkov S, Kulkova E (1938) On nosological place of scapula-peroneal amyotrophy. Zh Nevropat Psichiat 7: 11-20.

3. SEITZ D (1957) Nosological position of so-called scapulo-peroneal syndrome. Dtsch Z Nervenheilkd 175: 547-552.

4. Davidenkov SN, Dogel LV (1962) On scapuloperoneal form of progressive muscular atrophy, Notes on clinical neurology. Leningrad: Medgiz.

5. Becker PE (1967) New knowledge on genetics of the myopathies. Wien Klin Wochenschr 79: 402-405

6. Ricker K, Mertens HG (1968) The differential diagnosis of the myogenic (facio)scapulo-peroneal syndrome. Eur Neurol 1: 275-307.

7. Zellweger H, McCormick WF (1968) Scapuloperoneal dystrophy and scapuloperoneal atrophy. Helv Paediatr Acta 23: 643-649.

8. Serratrice G, Roux H, Aquaron R, Gambarelli D, Baret J (1969) Scapuloperoneal myopathies 14 cases including 8 with facial involvement. Sem Hop 45: $2678-2683$

9. In Hausmanowa-Petrusewicz I. (ed.): Muscle diseases. Warszawa: Panstwowy Zaklad Wydawnichw Lekarskich, p 179 (on Russian).

10. Kazakov VM (1971) Facio-scapulo-humeral myodystrophy (clinic and genetic); thesis Pavlov Medical Institute, Leningrad.

11. Kazakov VM, Bogorodinsky DK, Znoyko ZV, Skorometz AA (1974) The facioscapulo-limb (or the facioscapulohumeral) type of muscular dystrophy. Clinical and genetic study of 200 cases. Eur Neurol 11: 236-260.

12. Kazakov VM, Rudenko DI (1995) Clinical variability of facioscapulohumeral muscular dystrophy in Russia. Muscle Nerve Suppl 2: S85-S95.
13. Thomas PK, Schott GD, Morgan-Hughes JA (1975)Adult onset scapuloperonea myopathy. J Neurol Neurosurg Psychiatry 38: 1008-1015.

14. Carroll J.E. Facioscapulohumeral and scapuloperoneal syndromes (1979). In Vinken P.J., Bruyn G.W. (eds.): Handbook of clinical neurology. Diseases of muscle. Amsterdam: North-Holland, 40: 415 -431

15. Walton J.N., Gardner-Medwin D.(1981) Progressive muscular dystrophy and the myotonic disorders. In Walton J.N. (ed.): Disorders of voluntary muscle. 4th ed. Edinburgh: Churchill Livingstone, p.505

16. Walton J.N., Gardner-Medwin D. (1988) The facioscapulohumeral (FSH) muscular dystrophy. Autosomal dominant scapuloperoneal myopathy. In Walton J.N. (ed): Disorders of voluntary muscle. 5th ed. Edinburgh: Churchil Livingstone, 551-555.

17. Serratrice G, Pellissier JF, Pouget J, Gastaut JL, Cros D (1982) Scapuloperoneal syndromes. Rev Neurol (Paris) 138: 691-711.

18. Padberg GW (1982) Facioscapulohumeral disease (Thesis). Leiden: the Netherland University Leiden.

19. Rossi A, Giannini F, D’Amore I, Guazzi GC (1985) Landouzy-Dejerine disease: A progressive metameric muscular dystrophy of autosomal dominant inheritance with a variable intrafamilial phenotype expression. Acta Neurologica 2: 93-111.

20. Brooke M.H. (1986) Facioscapulohumeral dystrophy. Scapuloperonea dystrophy. In Brooke M.H. (ed,) A Clinician's view of neuromuscular diseases, 2nd ed. Baltimore: Williams \& Wilkins, pp. 158-175.

21. McKusick V.A. (1986) Scapuloperoneal syndrome, neurogenic, Kaeser type SCPNK. In OMIM- Online Mendelian Inheritance in Man, entry 181400, edit history: Carol 04/24/2012.

22. Swash M., Schwartz M.S. (1988) Muscular dystrophies. In Swash M., Schwartz M.S. (eds.) Neuromuscular diseases. A practical approach to diagnosis and management. 2nd ed. London: Springer-Verlag, p.292.

23. (1988) World Federation of Neurology Research Group on Neuromuscular Diseases. Classification of Neuromuscular Disorders, J Neurol Sci 86: 333-360.

24. Munsat TL, Serratrice G (1992) Facioscapulohumeral dystrophy and the scapuloperoneal syndromes, : Handbook of clinical neurology. Myopathies. Amsterdam: Elselvier Science 18: 161-77.

25. Brouwer OF (1993) Clinical and genetic aspects of facioscapulohumeral muscular dystrophy. Thesis. Leiden.

26. Jardine PE, Upadhyaya M, Maynard J, Harper P, Lunt PW (1994) A scapula onset muscular dystrophy without facial involvement: possible allelism with facioscapulohumeral muscular dystrophy. Neuromuscul Disord 4: 477-482.

27. Walton JN, Rowland LP, McLeod JG (1994) World Federation of Neurology Research Group on Neuromuscular Diseases. Classification of Neuromuscular Disorders, J Neurol Sci 124: 121-22.

28. Kazakov V (1995) History of the recognition and description of the facioscapulohumeral muscular dystrophy and on the priorities of Duchenne Erb, Landouzy and Dejerine. Acta Cardiomiol 79-84.

29. Griggs R.C., Mendel J. R., Miller R G. (1995) The muscular dystrophies. In Griggs R.C., Mendel J. R., Miller R. G. (eds): Evaluation and treatment of myopathies. Philadelphia: F.A. Davis, p. 127.

30. Tawil R, Myers GJ, Weiffenbach B, Griggs RC (1995) Scapuloperoneal syndromes. Absence of linkage to the 4q35 FSHD locus. Arch Neurol 52: 10691072

31. Rowland LP (1996) Molecular genetics and clinical neurology: Allelic heterogeneity, locus heterogeneity, gene-defining mutations and clinical syndromes, Acta Cardiomiol VIII: 41-51.

32. Wilhelmsen KC, Blake DM, Lynch T, Mabutas J, De Vera M, et al. (1996) Chromosome 12-linked autosomal dominant scapuloperoneal muscular dystrophy. Ann Neurol 39: 507-520.

33. Fisher J, Upadhyaya M (1997) Molecular genetics of facioscapulohumera muscular dystrophy (FSHD). Neuromuscul Disord 7: 55-62.

34. Bushby K.M.D. (1997) Autosomally inherited muscular dystrophy. In Rimoin D.L., Konnar J.M., Pyeritz R.E. (eds.): Emery and Rimoin's principles and practice of medical genetics. 3rd ed. New York: Churchill Livingstone, p. 2361.

35. Padberg G.W. (1998) Facioscapulohumeral muscular dystrophy. In Emery A.E.H. (ed): Neurumuscular disorders: Clinical and molecular genetics. Chichester: John Wiley \& Sons, p. 105 
36. Orrell R.W., Griggs R.C. (1999) Muscular dystrophies: Overview of clinical and molecular approaches. In Shapira A.H.V., Griggs R.C. (eds.): Muscle diseases. Boston: Butterworth Heinemann, pp.68-69.

37. Attarian S, Malzac P, Azulay JP (1999) Phenotype-genotype correlations studies in facioscapulohumeral muscular dystrophy, Acta Myol III: 57-58.

38. Neuromuscular Disease Center, Washington University School of Medicine, St Louis, MO 2003, 2012

39. Padberg G.W.( 2004) Facioscapulohumeral muscular dystrophy: a clinician's experience. In Upadhyaya M, Cooper D.N. (eds.): FSHD, Facioscapulohumera muscular dystrophy. Clinical medicine and molecular cell biology. London Bios Scientific, p. 41.

40. Zeevaert B, Sadzot B, Deprez M, Wang FC (2002) [Scapulo-peroneal weakness with tardive symptoms of facio-scapulo-humeral muscular dystrophy, connected to 4q35 chromosomal deletion]. Rev Neurol (Paris) 158: 1210-1213.

41. Pou-Serradell A. et al. (2004) Familial late-onset progressive of shoulder and ankle dorsiflexors weakness related to $4 \mathrm{q} 35$ chromosome deletion: Unusual phenotype of FSHD. Acta Myol.; XXIII: 63.

42. Kazakov VM, Bogorodinsky DK, Skorometz AA (1975) Myogenic scapuloperoneal syndrome - muscular dystrophy in the K. kindred. Reexamination of the K. family described for the first time by Oransky in 1927. Eur Neurol 13: 350-359.

43. Kazakov VM, Bogorodinsky DK, Skorometz AA (1976) The myogenic scapuloperoneal syndrome. Muscular dystrophy in the K. kindred: clinical study and genetics. Clin Genet 10: 41-50.

44. Kazalov VM, Rudenko DI, Skoromets AA (2008) Facioscapulohumeral muscular dystrophy and it connected with facioscapuloperoneal muscular dystrophy linked with chromosome $4 \mathrm{q} 35$. History, clinical, genetics and differential diagnosis. St. Petersburg: Polytechnics.

45. Kazakov V, Rudenko D, Kolynin V (2012) On nosological place of facioscapuloperoneal (or facioscapulolimb, type 2) 4q35-linked muscular dystrophy, FSHD International Research Consortium \& Research Planning Meeting, Abstracts, San Francisco, USA.

46. Kazakov V, Rudenko D, Kolynin V (2012) On nosological place of facioscapuloperoneal (or facioscapulolimb, type 2) 4q35-linked muscular dystrophy, Acta Myol.2012; XXXI: 161-162.

47. Sacconi S, Lemmers R, Lahaut P (2012) FSHD2 may act as (epi)genetic modifier for FSHD1. FSHD International Research Consortium \& Research Planning Meeting. Abstracts, p. 31. San Francisco, USA.

48. Sacconi S, Camaño P, de Greef JC, Lemmers RJ, Salviati L, et al. (2012) Patients with a phenotype consistent with facioscapulohumeral muscular dystrophy display genetic and epigenetic heterogeneity. J Med Genet 49: 41 46.

49. Kazakov VM (1992) On nosological place of FSH MD of Landouzy and Dejerine. Acta Cardiomiol IV: 11-18.

50. Kazakov VM, Rudenko DI (1995) The clinical peculiarities of facioscapulohumeral muscular dystrophy, FSH Watch 1995 2: 10-11

51. Kazakov VM (1998) On the name of FSHD and the famous discussion between Erb and Landouzy-Dejerine, once again, Acta Myol II: 72-74.

52. Kazakov V, Rudenko D, Sugita H, Goto K, Funakoshi M, et al. (2000) Facioscapulolimb muscular dystrophy, type 2 (FSLD2) (the same as a facioscapuloperoneal form of FSHD) in Russian families. Clinical aspects of the phenotype/genotype. Preliminary data. Oral presentation. The third International symposium on the cause and treatment of facioscapulohumeral muscular dystrophy. Abstracts. Bethesda, Maryland, USA.

53. Kazakov V, Rudenko D, Katsev H (2000) Facioscapulolimb muscular dystrophy (facioscapuloperoneal form of FSHD) in Russian families. Phenotype/genotype correlations. Acta Myol XIX: 69-79.

54. Kazakov VM, Rudenko DI, Katsev E (2001) Facioscapulolimb muscular dystrophy, type 2 (FSLD2) (the same as a facioscapuloperoneal form of FSHD) in Russian families. Clinical aspects of the phenotype/genotype. Preliminary data. FSH Watch 7: 61-63.

55. Kazakov $\vee$ (2001) Why did the heated discussion arise between Erb and Landouzy-Dejerine concerning the priority in describing the facio-scapulohumeral muscular dystrophy and what is the main reason for this famous discussion? Neuromuscul Disord 11: 421.

56. Kazakov V, Katsev E, Rudenko D (2002) Muscle uptake of 99m technetium dyphosphanates in patients with FSLD2 (the same disease as a facioscapuloperoneal form of FSHD), Acta Myol XXI: 25-30.

57. Kazakov V, Rudenko D (2002) FSHD in Russian families: pattern of muscle involvement, severity of the disease and DNA fragment size correlations. Poster Presentation. FSHD International Consortium Research Meeting. Abstracts. Baltimore, USA.

58. Kazakov V (2003) What is Davidenkov's scapuloperoneal amyotrophy: is it a myopathic entity or a neurogenic syndrome? What was Davidenkov's opinion concerning this knotty problem? Neuromuscul Disord 13: 91-92.

59. Kazakov V, Rudenko D (2003) About nosological place of the facioscapuloperoneal(or facio-scapulo-limb, type 2) autosomal dominan muscular dystrophy. Historical,clinical and molecular genetic study. Poster presentation. FSHD International Research Consortium Research Workshop Meeting. Abstracts, Los Angeles, CA, USA.

60. V. Kazakov, D. Rudenko. (2003) About nosological place of the facioscapuloperoneal (or facio-scapulo-limb, type 2) autosomal dominan muscular dystrophy. Historical, clinical and molecular genetic study. Poster presentation. FSHD International Research Consortium Research Workshop Meeting. Abstracts, p. 2. Los Angeles, CA, USA.

61. Kazakov V, Rudenko D, Pozdnyakov A, Kolynin V (2005) Autosomal dominant facio-scapulo-peroneal muscular dystrophy with 4q35 deletion: Is it possible independent form but is not a variant of facioscapulohumeral dystrophy. Report of two Russian families. International Workshop on FSHD, Abstracts, Salt Lake City, USA.

62. Kazakov V, Rudenko D, Pozdnyakov A, Kolynin V (2006) 4q35 linked autosoma dominant facioscapuloperoneal muscular dystrophy is probably an independen form but is not a variant of facioscapulohumeral muscular dystrophy. Abstract. Neuromuscul. Disord 16: S70 (Poster presentation on the Internat. Congr. on Neuromuscul. Diseases, 2-7 July 2006, Istanbul, Turkey)

63. Kazakov V, Rudenko D, Kolynin V (2006) About nosological place of the 4q35 linked autosomal dominant facioscapuloperoneal muscular dystrophy (FSPD) Historical, clinical and genetics study. Abstract. Neuromuscul. Disord 16: S70. (Poster presentation on the Internat. Congr. on Neuromuscul. Diseases, 2-7 July 2006, Istanbul, Turkey).

64. Kazakov V, Pozdnyakov A, Kolynin V, Rudenko D (2006) Muscle MRI findings in comparison with clinical muscle patterns in patients with $4 \mathrm{q} 35$ linked facioscapuloperoneal (the same disease as a facio-scapulo-limb type 2) autosomal dominant muscular dystrophy. Abstract. Neuromuscul. Disord 16: S70-S71. (Poster presentation on the Internat. Congr. on Neuromuscul. Diseases, 2-7 July 2006, Istanbul, Turkey).

65. Kazakov VM, Rudenko DI, Kolynin VO (2006) Clinical, CT and MRI peculiarities in 4 q35 linked facioscapuloperoneal muscular dystrophy. Report of two Russian families. FSHD International Research Consortium Research Workshop, Abstracts, p. 25. New Orleans, USA

66. Kazakov V.M., Kolynin V.O., Rudenko D.I., Pozdnyakov A.V. (2007) Lower limb muscles MRI findings with 4q35-linked facio-scapulo-limb, type 2 muscula dystrophy (FSLD2) (or facioscapuloperoneal dystrophy (FSPD). FSHD International Research Consortium. Abstract, p.24., San Diego, CA, USA.

67. Kazakov V, Rudenko D (2008) About the facioscapulolimb, type 2 (or the facioscapuloperoneal) autosomal dominant muscular dystrophy in the $\mathrm{K}$ kindred once again. Reexamination in 1993 of he famous K. kindred described for the first time by Oransky in 1927, then Davidenkov, Kulkova in 1938 and Kazakov et al. 1975, 1976. Clinical and molecular genetic study. FSHD International Research Consortium Meeting, Abstracts, p.26. Philadelphia USA.

68. Kazakov V, Rudenko D, Skorometz A, Kolynin V (2009) Scapuloperoneal muscular dystrophy is an independent variant of FSHD? Acta Myol 28: 103.

69. Kazakov V., Rudenko D. (2010) The facio-scapulo-limb, type 2 (or the facioscapuloperoneal) autosomal dominant muscular dystrophy. FSHD International Research Consortium \& Research Planning Meeting Abstract, $p$. 44. Boston, CA, USA.

70. Kazakov V., Rudenko D., Kolynin V. (2011) Facioscapuloperoneal (o facioscapulolimb, type 2) 4q35-linked muscular dystrophy. Abstracts, p. 28-29. FSH Society FSHD International Research Consortium \& Research Planning Meeting, Boston, USA.

71. Bregmann LE (1899) Ein casuistischer Beitrag zur progressiven Muskelatrophie. Dtsch Z Nervenheilk 14: 254-87.

72. Riese W (1922) Ueber familiare vererbare Dystrophien der Gesichtsmuskulatur. Dtsch Z Nervenheilk 75: 214-23. 
Citation: Kazakov VM (2013) About Clinical Heterogeneity of FSHD with Some Historical Remarks. Hereditary Genetics S1: 006. doi:10.4172/21611041.S1-006

73. Pearson K (1933) Two new pedigrees of muscular dystrophy. Ann Eugen (Lond) 5:179-191.

74. JUSTIN BESANCON L, PEQUIGNOT H, CONTAMIN F, DELAVIERRE P,
ROLLAND P (1964) MYOPATHY OF THE LANDOUZY-D'EJERINE TYPE. REPORT OF A HISTORICAL CASE. Sem Hop 40: 2990-2999.

75. Singleton MC (1967) Facioscapulohumeral dystrophy; report of a case. Phys Ther 47: 465-472.

This article was originally published in a special issue, Hereditary Disorders handled by Editor(s). Dr. X. Long Zheng, University of Pennsylvania, USA 